\title{
Optimized parallel tempering simulations of proteins
}

\author{
Simon Trebst \\ Computational Laboratory, Eidgenössische Technische Hochschule Zürich, CH-8092 Zürich, Switzerland; \\ Theoretische Physik, Eidgenössische Technische Hochschule Zürich, CH-8093 Zürich, Switzerland; \\ and Microsoft Research and Kavli Institute for Theoretical Physics, University of California, Santa Barbara, \\ California 93106 \\ Matthias Troyer \\ Theoretische Physik, Eidgenössische Technische Hochschule Zürich, CH-8093 Zürich, Switzerland \\ Ulrich H. E. Hansmann \\ Department of Physics, Michigan Technological University, Houghton, Michigan 49931 and \\ John-von-Neumann Institute for Computing, Forschungszentrum Jülich, D-52425 Jülich, Germany
}

(Received 6 February 2006; accepted 21 February 2006; published online 1 May 2006)

\begin{abstract}
We apply a recently developed adaptive algorithm that systematically improves the efficiency of parallel tempering or replica exchange methods in the numerical simulation of small proteins. Feedback iterations allow us to identify an optimal set of temperatures/replicas which are found to concentrate at the bottlenecks of the simulations. A measure of convergence for the equilibration of the parallel tempering algorithm is discussed. We test our algorithm by simulating the 36-residue villin headpiece subdomain HP-36 where we find a lowest-energy configuration with a root-mean-square deviation of less than $4 \AA$ to the experimentally determined structure. (C) 2006 American Institute of Physics. [DOI: 10.1063/1.2186639]
\end{abstract}

\section{INTRODUCTION}

Understanding the folding of proteins from computer simulations is a long-standing but still elusive goal in computational biology. The difficulties stem from the fact that proteins are only marginal stable. At room temperature the free energy difference between the biologically active and unfolded states is only of order $\approx 10 \mathrm{kcal} / \mathrm{mol}$. However, this small gap is due to cancellations of large energetic and entropic terms which pose two major challenges to numerical simulations. On one hand, one has to find a universal model that captures this delicate balance. On the other hand, the competing interactions necessarily lead to a rugged energy landscape that makes the exhaustive sampling of lowtemperature configurations a challenging computational task. In general, it has been hard to distinguish which of the two difficulties is the limiting factor in computational protein studies.

In this paper we address the second challenge and apply a powerful sampling technique that allows to efficiently explore a complex energy landscape by systematically shifting computational resources towards the bottlenecks of a simulation, ${ }^{1,2}$ which are typically in the vicinity of free energy barriers. We test our algorithm by simulating the 36residue villin headpiece subdomain HP-36. This molecule has raised considerable interest in computational biology $\mathrm{y}^{3,4}$ as it is one of the smallest proteins with well-defined secondary and tertiary structures ${ }^{5}$ but at the same time with 596 atoms still accessible to simulations. ${ }^{6}$ Its structure which was resolved by NMR analysis and deposited in the Protein Data Bank (PDB code 1vii) is shown in Fig. 1.

Recent computationally intensive investigations have studied this protein using molecular dynamics ${ }^{7}$ and parallel tempering ${ }^{8}$ techniques. While the former study reports room temperature configurations that are within $<4.0 \AA$ to the native structure, these randomly sampled configurations could not be singled out from the misfolded structures in a rigorous way. The latter study ${ }^{8}$ tries to identify the biologically active state as those configurations which minimize the energy functional of an implicit solvent model. However, despite considerable long simulation times low-energy configurations that resemble the experimentally determined one were found only with less than $20 \%$ frequency at $T=250 \mathrm{~K}$. These conformers differed still by root-mean-square deviations (RMSDs) of $\sim 4-6 \AA$ from the native structure and could also not be distinguished by their energies from that of the predominant misfolded structure. What leads to the discrepancy with the experiments? The authors of Ref. 8 argue that it is due to poor approximations of the simulated force field and especially the implicit solvent model. Indeed, configurations with a RMSD of $\approx 4 \AA$ have been found later with high frequency in simulations with a modified energy function. ${ }^{9}$ However, the alterations of the implicit solvent model are ad hoc and not universal, ${ }^{10}$ while the parameters of the original model were fitted against experimental data. On the other hand, the data of Ref. 8 could also indicate that despite large computational efforts the simulation has not thermalized and the correct equilibrium distribution of low-energy configurations has not yet been found.

Deciding between the two alternatives in the above example is especially important as parallel tempering ${ }^{11}$ (also known as replica exchange method) has recently become the simulation technique of choice in protein studies. ${ }^{12-14}$ The question can be reformulated as how does one gauge the efficiency of a parallel tempering run and ensures that the sampling is sufficiently long to ensure thermal equilibration? 


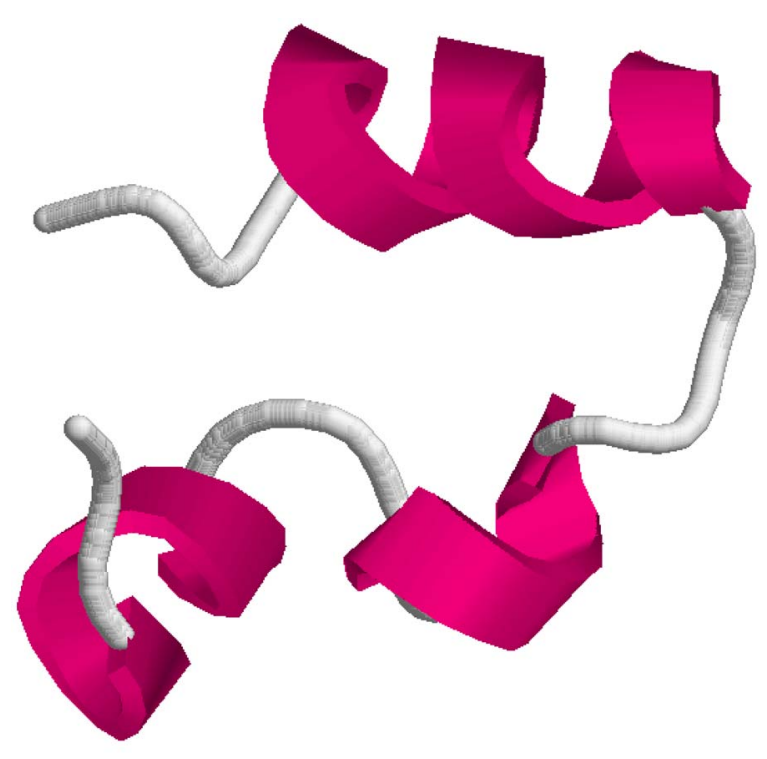

FIG. 1. NMR-derived structure of the 36-residue peptide HP-36 as deposited in the Protein Data Bank (1vii).

The present paper describes a measure for this purpose and discusses a protocol that allows one to optimize the performance of parallel tempering runs by finding the best temperature distribution. Using the enhanced parallel tempering protocol we demonstrate that the simulation of Ref. 8 had indeed not thermalized. On the contrary, we now find a dominant lowest-energy configuration that is within $<4.0 \AA$ to the native structure. This RMSD is comparable to the best ones found in previous molecular dynamics simulations ${ }^{7}$ with a different search technique and energy function, but our approach requires only $1 \%$ of their computational resources.

\section{ALGORITHM DESIGN}

In parallel tempering ${ }^{11}$ simulations $N$ noninteracting copies, or "replicas," of the protein are simultaneously simulated at a range of temperatures $\left\{T_{1}, T_{2}, \ldots, T_{N}\right\}$, e.g., by distributing the simulation over $N$ nodes of a parallel computer. After a fixed number of Monte Carlo sweeps (or a molecular dynamics run of a certain time) a sequence of swap moves, the exchange of two replicas at neighboring temperatures, $T_{i}$ and $T_{i+1}$, is suggested and accepted with a probability

$$
p\left(E_{i}, T_{i} \rightarrow E_{i+1}, T_{i+1}\right)=\min (1, \exp (\Delta \beta \Delta E)),
$$

where $\Delta \beta=1 / T_{i+1}-1 / T_{i}$ is the difference between the inverse temperatures and $\Delta E=E_{i+1}-E_{i}$ is the difference in energy of the two replicas. For a given replica the swap moves induce a random walk in temperature space that allows the replica to wander from low temperatures, where barriers in a complex energy landscape lead to long relaxation times, to high temperatures, where equilibration is rapid, and back. The convergence of a parallel tempering run is given by the relaxation at lowest temperature and can be gauged by the frequency of statistically independent visits at this temperature. A lower bound for this number is the rate of round trips $n_{\mathrm{rt}}$ between the lowest and highest temperatures, $T_{1}$ and $T_{N}$, respectively. An equivalent measure is the round-trip time $\tau_{1 N}$, i.e., the average time it takes a replica to move from lowest temperature $T_{1}$ to the highest temperature $T_{N}$ and back. It is this nonlocal measure in temperature space that one has to minimize in order to optimize a parallel tempering simulation. ${ }^{1,15}$ Commonly, it is assumed that equilibration is fastest if the local acceptance rate of swaps is the same for all pairs of neighboring temperatures $T_{i}$ and $T_{i+1} \cdot{ }^{16-20} \mathrm{Re}-$ cently, it has been shown that this assumption is misleading. ${ }^{2}$ Here we review the algorithm outlined in Ref. 2 and apply it to systematically optimize the temperature set used in our simulations in such a way that for each replica the number of round trips is maximized, and equilibration of the system at low temperatures thereby substantially improved.

We illustrate this approach by an example parallel tempering simulation of the 36-residue protein HP-36 in an allatom representation. Our approach is independent of the specific protein model; in order to compare our results with previous work we describe in our simulations the intramolecular interactions by the ECEPP/2 force field ${ }^{21}$

$$
\begin{aligned}
E_{\mathrm{ECEPP} / 2}= & E_{C}+E_{\mathrm{LJ}}+E_{\mathrm{hb}}+E_{\mathrm{tor}} \\
= & \sum_{(i, j)} \frac{332 q_{i} q_{j}}{\epsilon r_{i j}}+\sum_{(i, j)}\left(\frac{A_{i j}}{r_{i j}^{12}}-\frac{B_{i j}}{r_{i j}^{6}}\right) \\
& +\sum_{(i, j)}\left(\frac{C_{i j}}{r_{i j}^{12}}-\frac{D_{i j}}{r_{i j}^{10}}\right)+\sum_{l} U_{l}\left(1 \pm \cos \left(n_{l} \xi_{l}\right)\right),
\end{aligned}
$$

where $r_{i j}$ is the distance between the atoms $i$ and $j, \xi_{l}$ is the $l$ th torsion angle, and energies are measured in $\mathrm{kcal} / \mathrm{mol}$. The protein-solvent interactions are approximated by a solvent accessible surface term

$$
E_{\mathrm{solv}}=\sum_{i} \sigma_{i} A_{i}
$$

Here $A_{i}$ is the solvent accessible surface area of the $i$ th atom in a given configuration and $\sigma_{i}$ is a solvation parameter for the atom $i$. The solvent accessible surface areas $A_{i}$ are calculated by a variant of the double-cubic lattice method ${ }^{22}$ developed by Masuya. ${ }^{23}$ For the present investigation we use for the $\sigma_{i}$ the parameter set OONS of Ref. 24. Our implementation is based on the software package SMMP (simple molecular mechanics for proteins), ${ }^{25}$ which allowed us to distribute the simultaneous simulation of $N=20$ replicas on a Beowulf cluster with $2.2 \mathrm{GHz}$ Opteron processors. The initial temperature distribution for these replicas is listed in Table I. A sequence of swap moves between neighboring temperatures is attempted after each Monte Carlo sweep where a sweep consists of a series of Metropolis tests for each of the dihedral angles. Note that the implementation of the force field differs slightly from the one in Ref. 8 leading to (irrelevant) differences in the absolute energy values.

Our approach to optimize the simulated temperature set is inspired by a recently introduced adaptive broad-histogram algorithm $^{1}$ that maximizes the rate of round trips in energy space by shifting additional weight toward the bottlenecks of the simulation and has been outlined in the context of classical spin models in Ref. 2. The bottlenecks of the simulation are identified by measuring the local diffusivity of the simulated random walk. In the case of a parallel tempering run, 
TABLE I. Temperature sets used in the parallel tempering simulation of HP-36. Applying the feedback algorithm temperature points in the optimized temperature sets (iterations 2, 3, and 4) concentrate around the helix-coil transition around $500 \mathrm{~K}$. The temperature sets are also illustrated in Fig. 4.

\begin{tabular}{cccccccccccccccccccccc}
\hline \hline Iteration & \multicolumn{110}{c}{ Temperature set (K) } \\
\hline 1 & 250 & 275 & 300 & 325 & 350 & 375 & 400 & 425 & 450 & 500 & 550 & 600 & 650 & 700 & 750 & 800 & 850 & 900 & 950 & 1000 \\
2 & 250 & 295 & 326 & 349 & 371 & 395 & 424 & 446 & 464 & 482 & 499 & 514 & 528 & 543 & 559 & 577 & 595 & 628 & 693 & 1000 \\
3 & 250 & 326 & 359 & 385 & 411 & 434 & 452 & 467 & 480 & 491 & 501 & 510 & 519 & 527 & 536 & 546 & 560 & 578 & 619 & 1000 \\
4 & 250 & 314 & 358 & 381 & 402 & 423 & 444 & 461 & 474 & 484 & 494 & 502 & 511 & 519 & 529 & 540 & 554 & 576 & 670 & 1000 \\
\hline \hline
\end{tabular}

where we simulate a random walk in temperature space, this quantity is calculated by adding a label "up" or "down" to the replica that indicates which of the two extremal temperatures, $T_{1}$ or $T_{N}$, respectively, the replica has visited most recently. The label of a replica changes only when the replica visits the opposite extremum. For instance, the label of a replica with label up remains unchanged if the replica comes back to the lowest temperature $T_{1}$ but changes to down upon its first visit to $T_{N}$. For each temperature point in the temperature set $\left\{T_{i}\right\}$ we record two histograms $n_{\text {up }}\left(T_{i}\right)$ and $n_{\text {down }}\left(T_{i}\right)$. Before attempting a sequence of swap moves we increment at temperature $T_{i}$ that of the two histograms which has the label of the respective replica currently at temperature $T_{i}$. If a replica has not yet visited either of the two extremal temperatures, we increment neither of the histograms. For each temperature point this allows us to evaluate the average fraction of replicas which diffuse from the lowest to the highest temperature as

$$
f(T)=\frac{n_{\text {up }}(T)}{n_{\text {up }}(T)+n_{\text {down }}(T)} .
$$

In Fig. 2 this fraction is plotted for our parallel tempering simulations of HP-36 with an initial temperature distribution as listed in Table I.

The so-labeled replicas define a steady-state current from $T_{1}$ to $T_{N}$ that is proportional to the round-trip rate $n_{\mathrm{rt}}$ and therefore independent of temperature. To first order in the derivative this current is given by

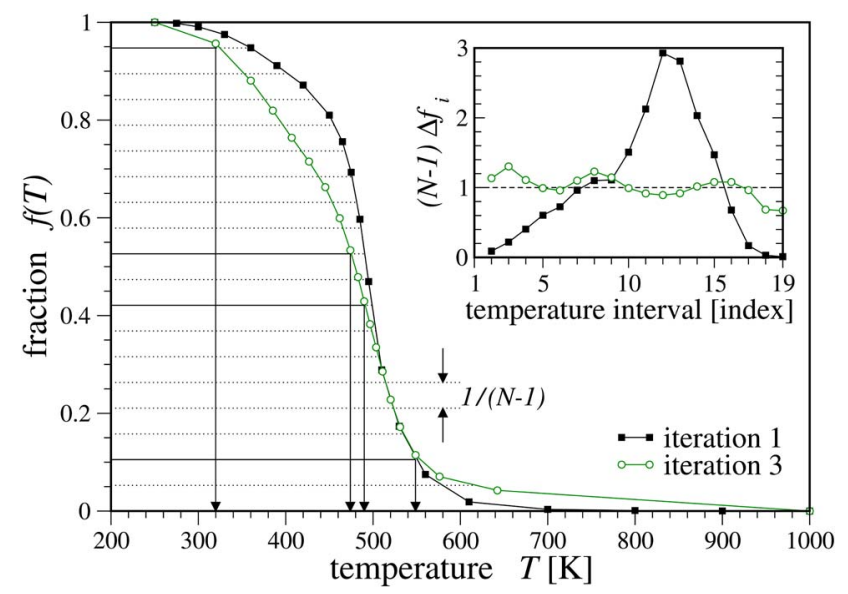

FIG. 2. Fraction of replicas diffusing from the lowest, $T_{1}=250 \mathrm{~K}$, to the highest, $T_{N}=1000 \mathrm{~K}$, temperature in a parallel tempering simulation of HP-36. For the optimized temperature set (iteration 3), the temperature points are distributed in such a way that the fraction shows a nearly constant decay $\Delta f_{i}=f\left(T_{i}\right)-f\left(T_{i+1}\right)=1 /(N-1)$ between adjacent temperature points, i.e., $\Delta f_{i}(N-1) \approx$ const.

$$
j=D(T) \eta(T) \frac{d f}{d T}
$$

where $D(T)$ is the local diffusivity at temperature $T$ and $\eta(T)$ is the probability distribution for a replica to reside at temperature $T$, where the temperature $T$ is now assumed to be a continuous variable (and not limited to the points of the current temperature set). For a given temperature set we approximate this probability distribution with a step function $\eta(T)=C / \Delta T$, where $\Delta T=T_{i+1}-T_{i}$ is the length of the temperature interval around temperature $T_{i}<T<T_{i+1}$ in the current temperature set. The normalization constant $C$ is chosen as

$$
\int_{T_{1}}^{T_{N}} \eta(T) d T=C \int_{T_{1}}^{T_{N}} \frac{d T}{\Delta T}=1
$$

Rearranging Eq. (5) gives a simple measure of the local diffusivity $D(T)$ :

$$
D(T) \sim \frac{\Delta T}{d f / d T}
$$

where we have dropped the normalization $C$ and the constant current $j$.

For the parallel tempering simulation of HP-36 this quantity is plotted in Fig. 3. The diffusivity shows a strong modulation along the simulated temperature range of 250-1000 K; note the logarithmic scale of the ordinate. A

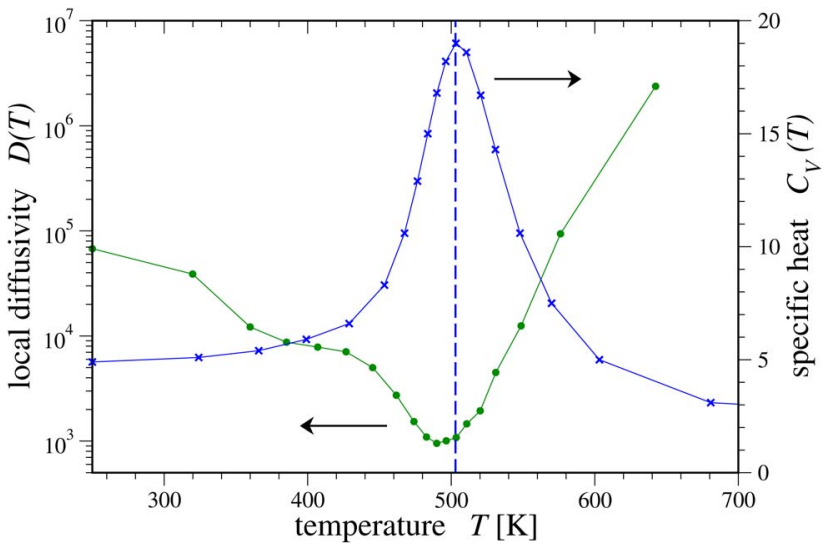

FIG. 3. Local diffusivity $D(T)$ (left ordinate) for a random walk in temperature space preformed by a replica in a parallel tempering simulation of the chicken villin headpiece subdomain HP-36 using the ECEPP/2 force field and an implicit solvent. The diffusivity shows a strong modulation with temperature; note the logarithmic scale of the left ordinate. A pronounced minimum in the local diffusivity occurs slightly below the helix-coil transition around $T \approx 500 \mathrm{~K}$ where the specific heat $C_{V}(T)$ (right ordinate) has a maximum (dashed line). 
pronounced minimum occurs around $T \approx 490 \mathrm{~K}$ where the diffusivity is suppressed by two orders of magnitude in comparison with the temperature range below $350 \mathrm{~K}$ and above $600 \mathrm{~K}$. This minimum in the diffusivity points to a severe bottleneck for the random walk in temperature space: Replicas can move back and forth in temperature rapidly below and above this bottleneck but experience a dramatic slowdown as they approach and pass through the temperature range around $490 \mathrm{~K}$. This behavior can be explained through a free energy barrier associated with a structural transition of the protein; the minimum in the local diffusivity is located slightly below the maximum of the specific heat at $T$ $\approx 500 \mathrm{~K}$ which is also plotted in Fig. 3. For HP-36 in the ECEPP/2 force field it has been shown that the position of this peak separates a high-temperature phase with extended unordered configurations from a low-temperature region that is characterized by high helical content of the molecule. ${ }^{8}$ Below this transition a shoulder in the measured local diffusivity points to a second bottleneck in the simulation for an extended range of temperatures $350 \leqslant T \leqslant 490 \mathrm{~K}$, possibly caused by competing low-energy configurations with high helical content. While the specific heat for this temperature range is slightly larger than in the high-temperature region above $600 \mathrm{~K}$, there is no characteristic feature similar to the progression of the local diffusivity. The local diffusivity is thus a more sensitive measure to identify bottlenecks in a parallel tempering simulation and to locate the multiple temperature scales dominating the folding process of a protein for a given force field.

In order to speed up equilibration we want to maximize the rate of round trips which each replica performs between the two extremal temperatures, or equivalently the diffusive current $j$, by varying the temperature set $\left\{T_{i}\right\}$ and thus the probability distribution $\eta(T)$, as discussed in Refs. 1 and 2 . Rearranging and integrating Eq. (5) this goal is achieved by minimizing the integral

$$
\frac{1}{j}=\int\left[\frac{1}{D(T) \eta(T)}+\lambda \eta(T)\right] d T,
$$

where we have added a Lagrange multiplier $\lambda$ which ensures that $\eta(T)$ remains a normalized probability distribution. Varying the probability distribution $\eta(T)$ the integrand in Eq. (8) is minimized for

$$
\eta^{\text {(opt) }}(T)=\frac{C^{\prime}}{\Delta T^{\prime}}=C^{\prime} \sqrt{\frac{1}{\Delta T} \frac{d f}{d T}} \sim \frac{1}{\sqrt{D(T)}},
$$

where the normalization $C^{\prime}$ is again chosen according to the normalization condition in Eq. (6). For the optimal temperature set the temperature points are thus rearranged in such a way that the probability distribution $\eta^{(\mathrm{opt})}(T)$ becomes inversely proportional to the square root of the local diffusivity. Measuring the local diffusivity $D(T)$ for an initial temperature set, we can determine the optimized probability distribution $\eta^{(\mathrm{opt})}(T)$ approximated as a step function in the original temperature set. The optimized temperature set $\left\{T_{i}^{\prime}\right\}$ is then found by choosing the $n$th temperature point $T_{n}^{\prime}$ such that

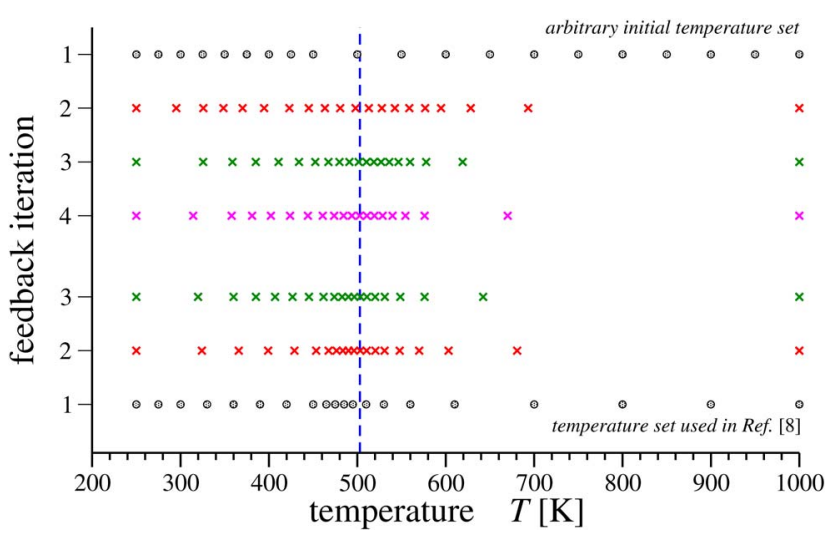

FIG. 4. Optimized temperature sets for a parallel tempering of HP-36 obtained by the feedback algorithm for two different initial temperature sets. Independent of the initial temperature set, the optimized temperature sets converge to a temperature set that concentrates temperatures in the vicinity of the helix-coil transition temperature around $T \approx 500 \mathrm{~K}$ (dashed line).

$$
\int_{T_{1}^{\prime}}^{T_{n}^{\prime}} \eta^{(\mathrm{opt})}(T) d T=n / N
$$

where $1<n<N$ and the two extremal temperatures $T_{1}^{\prime}=T_{1}$ and $T_{N}^{\prime}=T_{N}$ remain fixed. This feedback of the local diffusivity is then iterated for increasingly long simulation runs-in our simulations we double the number of swaps for subsequent feedback steps-until convergence of the optimized temperature set is found.

In our simulations we start with the arbitrary initial temperature set of Table I that similar to a geometric progression concentrates temperature points at low temperatures. Three feedback steps were performed: one after 100000 Monte Carlo (MC) sweeps, a second after further 200 000, sweeps, and a third one after additional 400000 sweeps. The iterated temperature sets are plotted in Fig. 4 and also listed in Table I. The feedback algorithm shifts computational resources towards the temperature of the helix-coil transition and temperature points in the optimized temperature sets concentrate around $T \approx 500 \mathrm{~K}$ where the measured local diffusivity is suppressed, see Fig. 4. In the derivation of the feedback procedure we have assumed that the local diffusivity is to leading order independent from the temperature set. A posteriori we can verify this assumption by demonstrating that the optimized temperature set is independent of the initial temperature set. To this end, we perform a second series of feedback optimization steps starting from the temperature set of Ref. 8. As illustrated in the lower half of Fig. 4 we indeed find that a very similar distribution is approached.

For the optimized temperature set the acceptance probabilities of replica swaps show a strong temperature dependence as illustrated in Fig. 5. This is a consequence of the concentration of temperature points around $T \approx 500 \mathrm{~K}$ in the optimized temperature set for HP-36. There the acceptance probabilities are found to be relatively high (around 80\%) while in the temperature regions below $350 \mathrm{~K}$ and above $600 \mathrm{~K}$ where temperature points have been thinned out the acceptance probabilities drop below some $40 \%$. The fact that for our optimized temperature set the acceptance probabilities vary with temperature contradicts various alternative ap- 


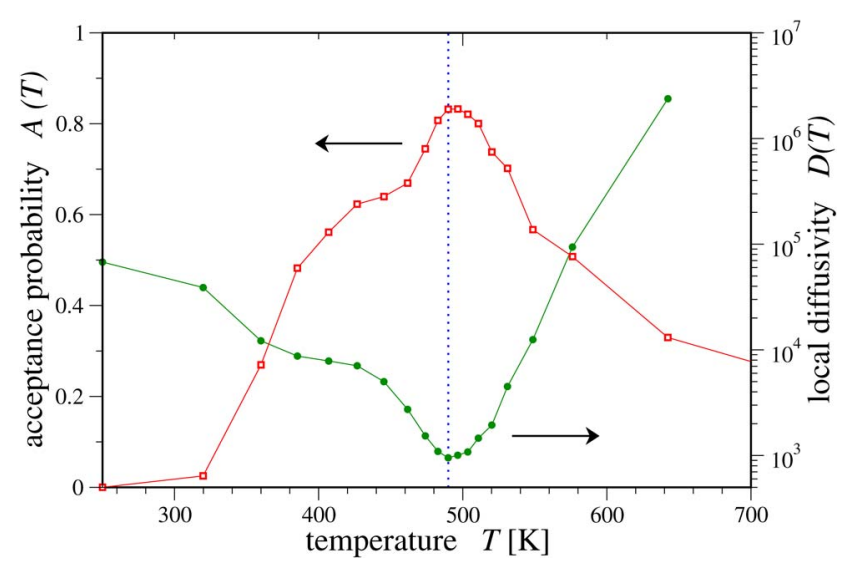

FIG. 5. Acceptance probabilities (open squares) of replica swaps in a parallel tempering simulation of HP-36 using the optimized temperature set illustrated in Fig. 4. The dependence of the acceptance probabilities on the temperature closely reflects the shape of the measured local diffusivity (filled circles). In the vicinity of the helix-coil transition temperature $T$ $\approx 500 \mathrm{~K}$ where the local diffusivity is strongly suppressed, the acceptance probabilities are highest due to the contraction of temperature points in the optimized temperature set. The dotted lines indicate the minimum in the local diffusivity.

proaches in the literature ${ }^{16-20}$ that aim at maximizing equilibration by choosing a temperature set where the acceptance probability of attempted swaps is independent of temperature.

\section{SIMULATION RESULTS}

The feedback iterations systematically optimize the temperature set which maximizes the efficiency of parallel tempering simulations. We now turn to the results obtained for our simulations of HP-36 and discuss the effects of the temperature reweighting. Though the parallel tempering simulations allow to evaluate thermodynamic quantities over a range of temperatures, here we focus on the properties of the configurations at the lowest temperatures. In Fig. 6 the radius of gyration $R_{\mathrm{gy}}$ which measures the compactness of a protein configuration is plotted for the lowest-energy configuration versus the number of Monte Carlo sweeps. For the initial

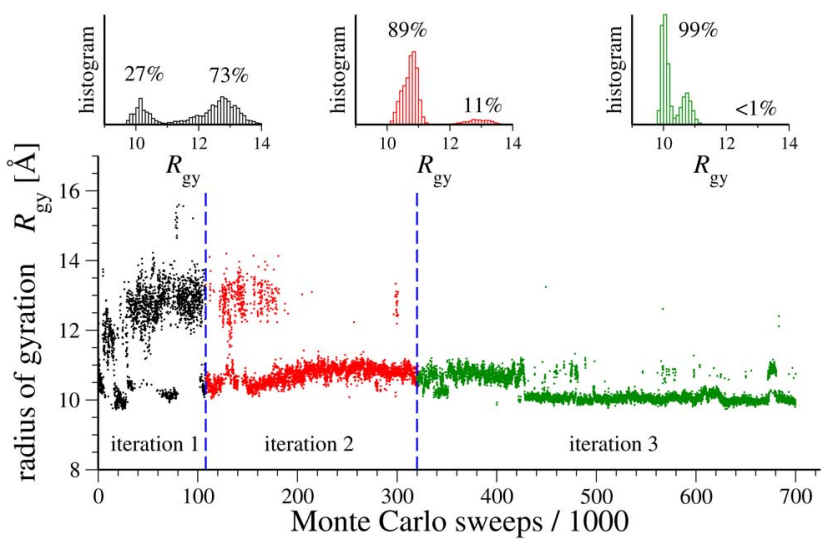

FIG. 6. Radius of gyration of the lowest-energy configuration in a parallel tempering simulation of HP-36 vs the number of Monte Carlo sweeps. The dashed lines indicate when the temperature set used in the simulation was redefined as illustrated in the upper half of Fig. 4. The insets show histograms of the radius of gyration for the three simulation parts.

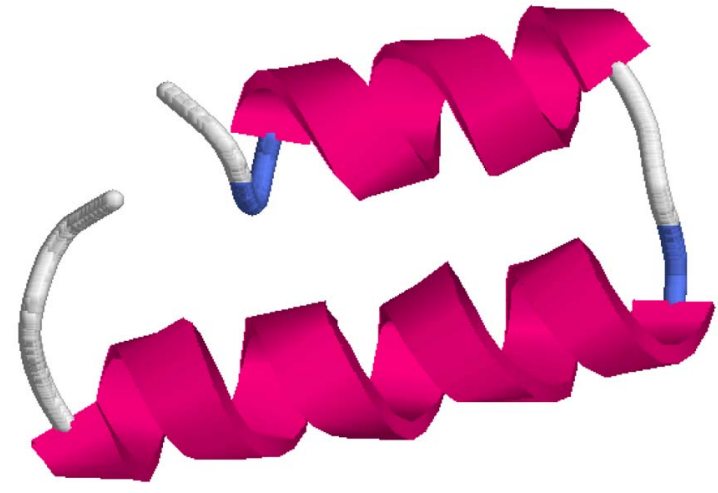

FIG. 7. Lowest-energy structure of HP-36 as obtained by an all-atom Monte Carlo simulation using the ECEPP/2 force field and an implicit solvent. The root-mean-square deviation of this structure to the PDB structure shown in Fig. 1 is $r_{\mathrm{RMSD}}=3.7 \AA$.

iteration the radius of gyration varies in a broad range of 10-14 $\AA$. A histogram of $R_{\mathrm{gy}}$ is plotted on top of the time series in Fig. 6 showing that two sets of configurations are found: one set with "compact" configurations characterized by a radius of gyration in the range of $10-11 \AA$ and "extended" configurations with a radius of gyration in the range of 12-14 A. Averaging over some 100000 MC sweeps in the first iteration we find that about $25 \%$ of the configurations are compact and a remaining $75 \%$ of extended configurations. Previous simulations ${ }^{8}$ with a total of $150000 \mathrm{MC}$ sweeps also reported the occurrence of these two sets of configurations. Similar to our case the extended configurations dominated, and only a small fraction of $20 \%$ of the configurations were compact. In the present study we continued the simulation after the first feedback step with an optimized temperature set for an additional $200000 \mathrm{MC}$ sweeps. The time series in Fig. 6 shows that as a consequence, the fraction of extended configurations in the lowest-energy configurations is significantly reduced and some $90 \%$ of the sampled lowest-energy configurations have a radius of gyration smaller than $11 \AA$. This ratio increases further to $99 \%$ for the final iteration with $400000 \mathrm{MC}$ sweeps after the second feedback step. While in the previous study equilibration at low temperatures was determined by analyzing the time series for thermodynamic observables such as the potential energy and convergence was found after some $100000 \mathrm{MC}$ sweeps, the discrepancy to the results presented here casts serious doubt whether an overall simulation time of some $150000 \mathrm{MC}$ sweeps and a suboptimal temperature set were sufficient to reach full equilibration. The long relaxation times in our example indicate that even with a sophisticated technique such as parallel tempering, the simulation times have to be considerably longer than commonly assumed. In order to assure equilibration at lowest temperature the number of round trip times should be at least $n_{\mathrm{rt}} \approx 10$.

To probe whether our simulations allow a structural prediction of the true ground state configuration we track the configuration with the overall lowest energy in the simulation and compare it to the Protein Data Bank structure of HP-36 (PDB code 1vii). The lowest-energy configuration obtained in our simulation is illustrated in Fig. 7. Despite the fact that in this structure the two N-terminal helices merged 
to one long helix (compromising residues 5-21) that tightly packs to the C-terminal helix, its RMSD to the PDB structure is only $r_{\mathrm{RMSD}}=3.7 \AA$. This value is substantially lower than in the structures with an RMSD of $r_{\mathrm{RMSD}} \approx 5.8-6.0 \AA$ previously obtained by molecular dynamics simulations, ${ }^{6}$ Monte Carlo simulations, ${ }^{8,9}$ and optimization techniques. ${ }^{26}$ A structure with comparable RMSD of $r_{\mathrm{RMSD}} \approx 3.5 \AA$ has been obtained by large-scale molecular dynamics simulations. ${ }^{7}$ However, in those simulations the best matching structure was found by comparing all sampled configurations along multiple trajectories to the PDB structure, while in our simulations the optimal structure is singled out as the one with the lowest energy. In addition, our simulations consumed only about $1 \%$ of the computing time resources (about 1000 CPU years) used in Ref. 7.

\section{CONCLUSIONS}

In conclusion, we have applied a powerful feedback algorithm for the numerical simulation of proteins that allows to allocate computational resources in a parallel tempering simulation so that equilibration at all temperatures is considerably improved. By tracking the diffusion of replicas in temperature space we have identified the bottlenecks of a simulation, typically in the vicinity of the folding transition. Feeding back this information we obtain an optimal temperature set that concentrates temperature points at these bottlenecks. Our algorithm differs from previous approaches that aim at maximizing equilibration by considering the local acceptance probabilities of replica exchange moves. In contrast we find that for the optimal temperature set acceptance probabilities for such swap moves show a strong temperature dependence. Applying the optimized parallel tempering technique to the simulation of the 36-residue protein HP-36, we find a dominant low-energy configuration with less than $4 \AA$ root-mean-square distance from the native structure within a fraction of the computing time consumed by highperformance molecular dynamics simulations. On the other hand, in a previous work ${ }^{8}$ without an optimal temperature distribution, we found as dominating low-energy configuration an extended structure which differs from the PDB structure by a RMSD of $8.0 \AA$.

We note, however, that the energy difference between our compact, lowest-energy configuration and the extended structure of Ref. 8 is only $\approx 10 \mathrm{kcal} / \mathrm{mol}$ (for the minimized configurations). On the other hand, the energy of our lowestenergy configuration is $100 \mathrm{kcal} / \mathrm{mol}$ lower than that of the (minimized) PDB structure from which (despite the small RMSD) it still differs considerably. Hence, while our results appear to be closer to the experimental results than previous simulations, they still demonstrate the limitations on protein simulations that are inherent in present energy functions. The extremely long relaxation times indicate the existence of spurious minima that should be absent in the folding funnel of fast folding proteins such as the villain headpiece. Unveiling these limitations in the energy functions and their underlying causes requires optimized simulation techniques such as the one applied in the present paper.

\section{ACKNOWLEDGMENTS}

The authors thank A. Laio and B. Zagrovic for stimulating discussions, as well as D. A. Huse and H. G. Katzgraber for fruitful discussions on the technical aspects of this manuscript. One of the authors (S.T.) acknowledges support by the Swiss National Science Foundation and another author (U.H.E.H.) by a research grant (CHE-9981874) of the National Science Foundation (USA).

${ }^{1}$ S. Trebst, D. A. Huse, and M. Troyer, Phys. Rev. E 70, 046701 (2004).

${ }^{2}$ H. G. Katzgraber, S. Trebst, D. A. Huse, and M. Troyer, J. Stat. Mech. P03018 (2006).

${ }^{3}$ M. Y. Shen and K. F. Freed, Proteins 49, 439 (2002).

${ }^{4}$ D. R. Ripoli, J. A. Vila, and H. A. Scheraga, J. Mol. Biol. 339, 915 (2004).

${ }^{5}$ C. J. McKnight, D. S. Doehring, P. T. Matsudaria, and P. S. Kim, J. Mol. Biol. 260, 126 (1996).

${ }^{6}$ Y. Duan and P. A. Kollman, Science 282, 740 (1998).

${ }^{7}$ B. Zagrovic et al., J. Mol. Biol. 323, $153 \mathrm{i}$ (2002).

${ }^{8}$ C.-Y. Lin, C.-K. Hu, and U. H. E. Hansmann, Proteins: Struct., Funct., Genet. 52, 436 (2003).

${ }^{9}$ U. H. E. Hansmann, Phys. Rev. E 70, 012902 (2004).

${ }^{10}$ W. Kwak and U. H. E. Hansmann, Phys. Rev. Lett. 95, 138102 (2005).

${ }^{11}$ K. Hukushima and K. Nemoto, J. Phys. Soc. Jpn. 65, 1604 (1996); G. J. Geyer, Stat. Sci. 7, 437 (1992).

${ }^{12}$ U. H. E. Hansmann, Chem. Phys. Lett. 281, 140 (1997).

${ }^{13}$ U. H. E. Hansmann and Y. Okamoto, Curr. Opin. Struct. Biol. 9, 177 (1999).

${ }^{14}$ U. H. E. Hansmann, Comput. Sci. Eng. 5, 64 (2003).

${ }^{15}$ P. Dayal et al., Phys. Rev. Lett. 92, 097201 (2004).

${ }^{16}$ D. A. Kofke, J. Chem. Phys. 117, 6911 (2004).

${ }^{17}$ C. Predescu, M. Predescu, and C. Ciabanu, J. Chem. Phys. 120, 4119 (2004).

${ }^{18}$ A. Kone and D. A. Kofke, J. Chem. Phys. 122, 20610 (2005).

${ }^{19}$ N. Rathore, M. Chopra, and J. J. de Pablo, J. Chem. Phys. 122, 024111 (2005).

${ }^{20}$ C. Predescu, M. Predescu, and C. Ciabanu, J. Phys. Chem. B 109, 4189 (2005).

${ }^{21}$ M. J. Sippl, G. Némethy, and H. A. Sheraga, J. Phys. Chem. 88, 6231 (1994).

${ }^{22}$ F. Eisenhaber, P. Lijnzaad, P. Argos, C. Sander, and M. Scharf, J. Comput. Chem. 16, 273 (1995).

${ }^{23} \mathrm{M}$. Masuya, NSOL-a numerical calculation program of molecular surface area, volume, and solvation energy, computing and communications center, Kagoshima University 1999-2003; http://biocomputing.cc/nsol

${ }^{24}$ T. Ooi, M. Obatake, G. Nemethy, and H. A. Scheraga, Proc. Natl. Acad. Sci. U.S.A. 8, 3086 (1987).

${ }^{25}$ F. Eisenmenger, U. H. E. Hansmann, Sh. Hayryan, and C.-K. Hu, Comput. Phys. Commun. 138, 192 (2001).

${ }^{26}$ U. H. E. Hansmann and L. T. Wille, Phys. Rev. Lett. 88, 068105 (2002). 